This document is the accepted manuscript version of the following article:

Lee, P. K. H., Men, Y., Wang, S., He, J., \& Alvarez-Cohen, L. (2015). Development of a fluorescence-activated cel1 sorting method coupled with whole genome amplification to analyze minority and trace Dehalococcoides genomes in microbial communities. Environmental Science and Technology, 49(3), 1585-1593. https://doi.org/10.1021/es503888y

\title{
Development of a Fluorescence-Activated Cell Sorting Method Coupled with Whole Genome Amplification To Analyze Minority and Trace Dehalococcoides Genomes in Microbial Communities
}

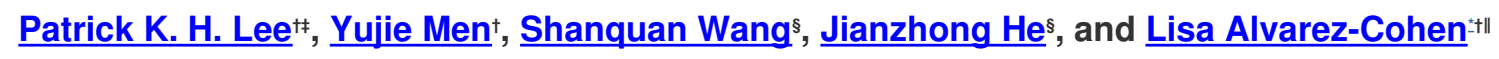

+ Department of Civil and Environmental Engineering, University of California, Berkeley, California 94720, United States

₹ School of Energy and Environment, City University of Hong Kong, Hong Kong

\& Division of Environmental Science and Engineering, National University of Singapore, Singapore 117576

" Earth Sciences Division, Lawrence Berkeley National Laboratory, Berkeley, California 94720, United States

*Phone: (510)643-5969; fax: (510) 642-7483; e-mail: alvarez@ce.berkeley.edu.

DOI: $10.1021 /$ es503888y

Publication Date (Web): January 7, 2015

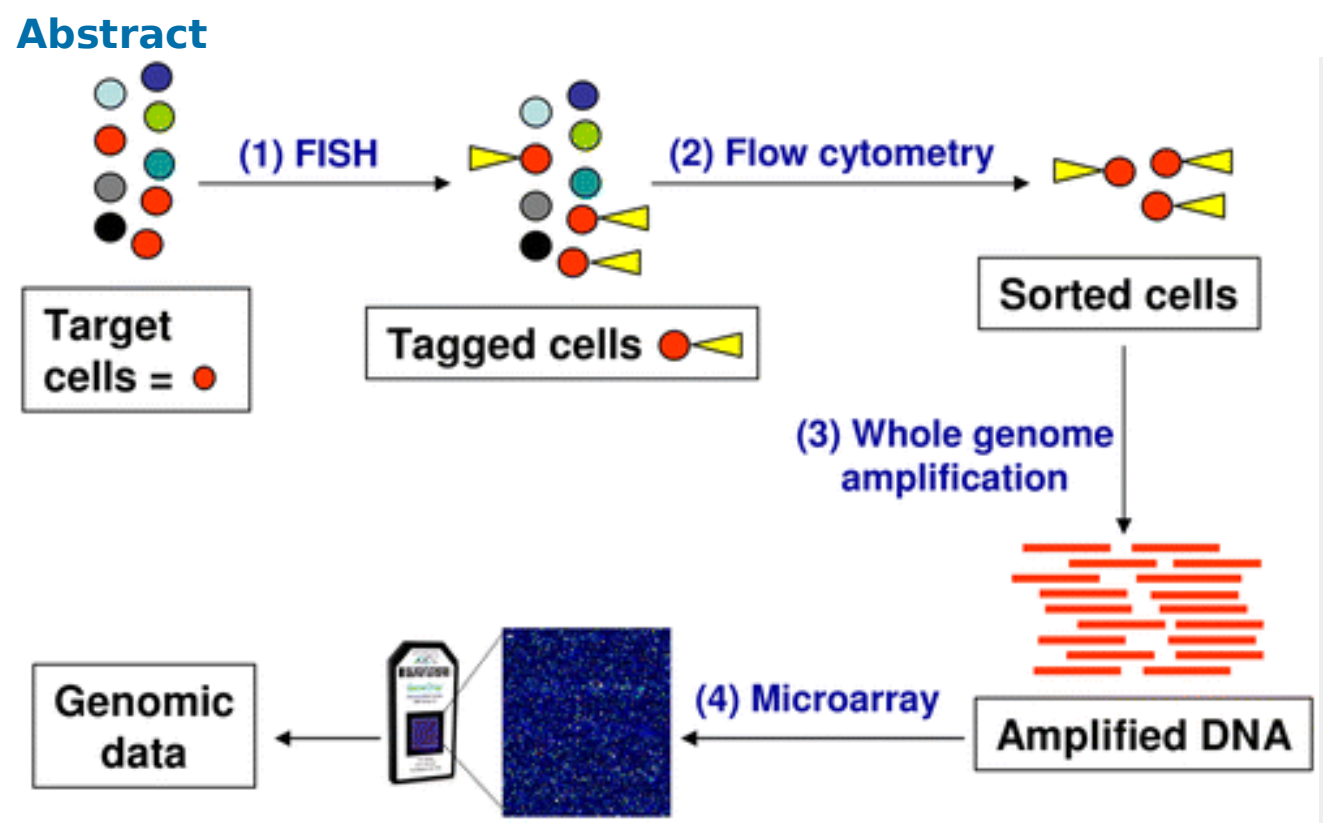

Dehalococcoides mccartyi are functionally important bacteria that catalyze the reductive dechlorination of chlorinated ethenes. However, these anaerobic bacteria are fastidious to isolate, making downstream genomic characterization challenging. In order to facilitate genomic analysis, a fluorescence-activated cell sorting (FACS) method was developed in this study to separate $D$. mccartyi cells from a microbial community, and the DNA of the isolated cells was processed by whole genome amplification (WGA) and hybridized onto a D. mccartyi microarray for comparative genomics against four sequenced strains. First, FACS was successfully applied to a $D$.

mccartyiisolate as positive control, and then microarray results verified that WGA from $10^{6}$ cells or $\sim 1 \mathrm{ng}$ of genomic DNA yielded high-quality coverage detecting nearly all genes across the genome. As expected, some inter- and intrasample variability in WGA was observed, but these biases were minimized by performing multiple parallel amplifications. Subsequent application of the FACS and WGA protocols to two enrichment cultures containing $\sim 10 \%$ and $\sim 1 \%$ D. mccartyi cells successfully enabled genomic analysis. As proof of concept, this study demonstrates that coupling FACS with WGA and microarrays is a promising tool to expedite genomic characterization of target strains in environmental communities where the relative concentrations are low. 


\section{Introduction}

Genomic analysis of environmental microbial communities that perform important functional activities involving only a small minority of the community members can be extremely challenging. One important example is subsurface microbial communities involved in the in situ bioremediation of chlorinated ethenes. Chlorinated ethenes are known and suspected carcinogens whose improper storage and disposal has resulted in widespread groundwater contamination.(1)Fortunately, Dehalococcoides mccartyi bacteria can reductively dechlorinate these compounds to the innocuous product ethene,(2) making in situ bioremediation a promising approach for environmental cleanup efforts.(3) One of the first priorities when applying an in situ bioremediation approach is to evaluate the metabolic functions of the indigenous subsurface microbial population. Traditionally this has been accomplished using culturing approaches in the laboratory by establishing microcosms using indigenous soil and/or groundwater samples.(2) However, culturing approaches are time-consuming and labor intensive; therefore, different cultureindependent methods based on polymerase chain reaction (PCR) or quantitative PCR (qPCR) with specific primer sets have also been developed to target phylogenetic markers of $D$. mccartyi and genes that are known to encode enzymes for catalyzing reductive dechlorination of specific chlorinated ethenes.(4-12)

Ideally, all genes across the genomes of each $D$. mccartyi present would be queried to obtain a comprehensive assessment of metabolic potentials, $(3,13)$ but conventional PCR-based approaches are poorly suited for this purpose due to low throughput. In contrast, metagenomic sequencing and microarray analysis are two highthroughput culture-independent methods that depend on de novo sequencing and comparison against known sequences, respectively, to rapidly and efficiently analyze the overall gene content of an unknown sample.(14,

15) Recently, a microarray targeting the $>6000$ genes from four sequenced $D$. mccartyi genomes(16-18) has been designed and successfully applied to query the gene content of unsequenced isolates or $D$. mccartyi-containing enrichments.(19) However, application of both metagenomic sequencing and microarray approaches that target specific subpopulations within complex microbial communities can be inherently challenging as the target genomes can be masked by other sequences, especially if the relative concentration of the target organisms is low. 20 , 21) Therefore, in order to enhance the resolution of genomic analysis, fluorescence-activated cell sorting (FACS) techniques followed by whole genome amplification (WGA) have been developed in recent years to separate and analyze specific organisms within complex communities.(22-25)

The aim of this study was to establish a protocol that uses FACS to separate and recover D. mccartyi populations from microbial communities. Because microarrays are less time-consuming and expensive than high-coverage nextgeneration sequencing, and therefore can be applied more routinely and repetitively, genomic DNA (gDNA) from the sorted cells were amplified and applied to microarrays targeting $D$. mccartyi genomes(19) to rapidly perform comparative genomics. The approach of coupling FACS with WGA and analysis by microarray was first demonstrated with a pure culture and later with two enrichment cultures containing $\sim 10 \%$ and $\sim 1 \%$ D. mccartyi as proof of concept to pave the way for application in environmental samples for diagnostic purposes in the future. 


\section{Materials and Methods}

\section{Bacterial Strain and Enrichment Cultures}

Dehalococcoides mccartyi strain 195(26, 27) (strain 195) and enrichment cultures GW2 and AD14 containing 10\% and $\sim 1 \%$ D. mccartyi cells, respectively, were all grown in defined medium as described previously.(28,

29) Enrichment GW2 was established using groundwater from a trichloroethene (TCE)-contaminated site in New Jersey $(30)$ and enrichment AD14 was developed using sludge from an anaerobic digester of a pesticide factory in Gehua (Hubei Province, China).(31) Both use lactate as electron donor and carbon source and dechlorinate TCE to vinyl chloride (VC) or ethene. $(30,31)$ The concentrations of tce $A, v c r A$, and $16 \mathrm{~S}$ rRNA genes of $D$. mccartyi, and bacteria within the enrichments were determined according to a previously described qPCR method.(32)

\section{Fluorescence In Situ Hybridization (FISH)}

Actively dechlorinating cells from strain $195\left(\sim 10^{\circ}\right)$ or the enrichment cultures $\left(\sim 10^{10}\right.$ total cells $)$ were harvested by centrifugation $\left(21000 \mathrm{~g}, 3 \mathrm{~min}, 4^{\circ} \mathrm{C}\right.$ ), the supernatant removed, and cells resuspended in $200 \mu \mathrm{L}$ of $1 \times$ Phosphate Buffered Saline (PBS) (Gibco, NY). Cells were fixed with $600 \mu \mathrm{L}$ of $4 \%$ paraformaldehyde (w/v in $1 \times$ PBS) at $4{ }^{\circ} \mathrm{C}$ for $13 \mathrm{~h}$. The fixed cells were washed three times with $800 \mu \mathrm{L}$ of $1 \times$ PBS and resuspended in $180 \mu \mathrm{L}$ of hybridization buffer prepared according to a described protocol with 30\% formamide.(33) Duplicate cultures were analyzed for each experiment and three samples were prepared from each culture for the hybridization of different probes. Two samples were used as negative controls where no probe or the nonspecific probe Non338(34) (5'-

ACTCCTACGGGAGGCAGC-3') was added and the third was incubated with a published $D$. mccartyi-specific probe (5'-AGCTCCAGTTCRCACTGTTG-3') targeting 16S rRNA.(33) The no probe and Non338 probe controls were used to evaluate the extent of autofluorescence and nonspecific bindings, respectively. Probes labeled with Alexa Fluor 488 were synthesized by Invitrogen (Carlsbad, CA) and added to a final concentration of $5 \mathrm{ng} / \mu \mathrm{L}$ in the hybridization reaction.(22) Samples were incubated at $37^{\circ} \mathrm{C}$ in the dark for $2 \mathrm{~h}$ and then stained with $20 \mathrm{ng} / \mu \mathrm{L}(33)$ of the DNAintercalating dye 4',6-diamidino-2-phenylindole (DAPI; Invitrogen, Carlsbad, CA) for 15 min. After hybridization, cells were pelleted $\left(21000 \mathrm{~g}, 10 \mathrm{~min}, 25^{\circ} \mathrm{C}\right)$ and incubated with $500 \mu \mathrm{L}$ of prewarmed hybridization buffer at $37^{\circ} \mathrm{C}$ for 20 $\min$. Subsequently, cells were pelleted and washed with $500 \mu \mathrm{L}$ of prewarmed wash buffer(33) at $48^{\circ} \mathrm{C}$ for 20 min. Finally, cells were resuspended and homogenized in $50 \mu \mathrm{L}$ of $1 \times$ PBS for microscopic observations and cell sorting.

Cells were observed using a Zeiss Axiolmager M1 epifluorescence microscope (Thornwood, NY) and a 100× oil immersion objective with the appropriate filter set (DAPI, $358 \mathrm{~nm}$ (excitation)/461 nm (emission); Alexa Fluor 488, $495 \mathrm{~nm}$ (excitation)/519 nm (emission)) at the UC Berkeley Biological Imaging Facility. Images were captured separately from the same examining field with an Orca-03 CCD digital camera (Hamamatsu, Japan) and an exposure time of $0.25 \mathrm{~s}$ for both DAPI and Alexa Fluor 488. Images were viewed using the iVision software (BioVision Technologies, PA). All samples were viewed with microscopy prior to cell sorting.

\section{Flow Cytometry and Cell Sorting}


Cell sorting was performed on a MoFlo high speed flow cytometer (Dako-Cytomation, Carpinteria, CA) at the UC Berkeley Flow Cytometry Facility. The instrument was stringently sterilized prior to each run. Forward and side light scattering as well as the Alexa Fluor 488 fluorescence of microbial cells were measured. The sorting gate was determined using the two negative controls with no probe and the nonspecific probe in conjunction with the sample labeled with the $D$. mccartyi-specific probe. Cells exhibiting a fluorescence signal above the cutoff were gated and collected into $1.5 \mathrm{~mL}$ centrifuge tubes.

\section{Whole Genome Amplification (WGA)}

Samples containing the desired number of sorted cells were pelleted at $21000 \mathrm{~g}, 20 \mathrm{~min}, 4^{\circ} \mathrm{C}$ and the supernatant removed. gDNA was amplified using a REPLI-g midi kit (Qiagen, Valencia, CA) according to the manufacturer's instructions for single cell amplification. Lysis buffer D3 was used and an incubation step at $65{ }^{\circ} \mathrm{C}$ for 5 min was followed by incubation on ice. The amplification reaction lasted $16 \mathrm{~h}$ at $30{ }^{\circ} \mathrm{C}$. The amplified DNA was cleaned-up using a QIAamp DNA Mini Kit (Qiagen, Valencia, CA) according to a protocol developed by the manufacturer for REPLI-g amplified DNA. The amplified DNA was quantified with a fluorometer (Turner BioSystems, Sunnyvale, CA) using the PicoGreen double-stranded DNA quantitation kit (Invitrogen, Carlsbad, CA) according to the manufacturer's instructions.

\section{Microarray Analysis}

Triplicate samples of $1 \mu \mathrm{g}$ amplified DNA or gDNA without amplification from cultures were prepared for microarray hybridization according to previously described protocols.(19) The resulting data (Table S1 of the Supporting Information (SI)) were normalized and analyzed according to previously described procedures.(19) Briefly, for a gene to be considered "present", the corresponding probe set across all three replicate samples had to have signal intensities greater than 140 and a $P$ value less than 0.05 . The minimum signal requirement was not applied to the amplified DNA from enrichment GW2 as the overall microarray signal intensity was lower, but probes must satisfy the statistical significance threshold $(P<0.05)$. The microarray data analyzed in this study were deposited in the NCBI's Gene Expression Omnibus database under accession number GSE54040.

\section{Results}

\section{Method Validation with Isolate}

The applicability of FACS was first tested with the isolate strain 195 as positive control. Applying a $D$. mccartyispecific probe targeting the $16 \mathrm{~S}$ rRNA, (33) we verified that the fluorescence signals were detectable with microscopic observations and flow cytometry and were significantly stronger than the autofluorescence and nonspecific binding controls (Figure 1 ). Previous results in the literature have suggested that at least $1 \mathrm{ng}$ of DNA is necessary for high-fidelity WGA.(22, 35-37)Because $D$. mccartyi genomes are relatively small $(\sim 1.5 \mathrm{Mbp}), 10^{6} \mathrm{strain}$ 195 cells were sorted to obtain 1 ng of starting DNA for WGA. 


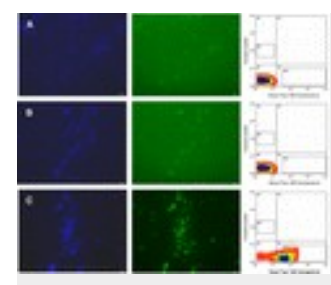

Figure 1. DAPI (left column) and Alexa Fluor 488 (middle column) fluorescence microscopy of the same examining field, and flow cytometry analysis (right column) of strain 195. The scale bar represents $5 \mu \mathrm{m}$. Each row indicates a sample: (A) no probe negative control, (B) Non338 negative control and (C) D. mccartyi-specific probe. Each flow cytometry plot contains $\sim 120000$ events. Cells from the $D$. mccartyi-specific probe labeled samples had stronger green fluorescence signals than the negative controls as observed in microscopy and cells with signals within the R23 gate in flow cytometry were sorted for downstream analyses. The R23 gate was chosen because cells from the two negative controls had fluorescence signals below this range. The other gates ( $\mathrm{R}$ regions) were not considered.

Using a commercially available WGA kit, two identically sorted samples from the same subculture of strain 195 were amplified and hybridized onto microarrays (Figure $2 \mathrm{~A}$ ). Based on the hybridization results, $1 \mathrm{ng}$ of starting DNA yielded high quality amplification. In fact, with the exception of only four genes (DET1089, DET1091, DET1092, DET1094) in one of the two samples, all genes across the genome were designated present with genes near genome positions 1100 and 1400 noticeably under-represented compared to the other gDNA (Figures $\underline{2} A$ and $B$ ). Although nearly all genes across the genome were amplified and detected, intrasample amplification variability was observed as the coefficient of variation (CV) across all probe sets for gDNA of strain 195 (Figure $2 \mathrm{~B}$ ) was only $15.9 \%$, but it was $35.7 \%$ and $33.7 \%$ for the two WGA samples. Besides intrasample variability, differences in signal intensity for duplicate samples also illustrated intersample variability, mostly on a region-to-region basis across the genome (Figure $\underline{2} \mathrm{C}$ ). In order to minimize amplification biases, two samples were sorted from each of the duplicate cultures and equal masses of the amplified DNA were pooled prior to microarray analysis (Figure 2D). With this pooling procedure, the CV of probe sets improved slightly to $30.9 \%$ and all genes were positively detected. This averaging process improved the signal intensity near genome position 1100 and elsewhere when compared to results from an individual amplification (Figures $\underline{2} \mathrm{~A}$ and $\mathrm{D}$ ). Four WGA samples from duplicate cultures were subsequently prepared and pooled for each experiment in this study.

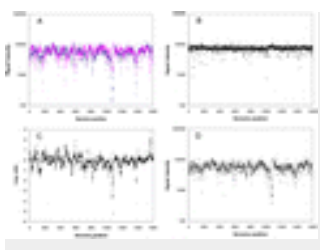

Figure 2. (A) Microarray signal intensity of two (pink and dark blue) independently sorted and WGA samples ( $10^{6}$ cells) from the same subculture of strain 195. For each panel, a data point represents a gene of strain 195 arranged according to its location in the genome from DET0001 going left to right. (B) Signal intensity from hybridization of gDNA of strain 195 (original data from Lee et al.(19)). (C) Ratio of signal intensity between the two amplified samples shown in (A). (D) Signal intensity obtained after pooling equal proportion of four independently sorted and WGA DNA from duplicate cultures.

\section{Application of FACS-WGA to D. mccartyi-Containing Enrichments}


After verifying the FACS-WGA method with a pure culture, the protocol was applied to two enrichment cultures containing different proportions of $D$. mccartyi cells (Figure $\underline{3}$ and Figure $\mathrm{S} 1$ of the $\mathrm{SI}$ ). For the flow cytometry separation, the fluorescence of cells labeled with the $D$. mccartyi-specific probe was distinctly different from the negative controls, enabling the sorting of $10^{6} \mathrm{D}$. mccartyi cells from both enrichments. In addition, $10^{5} \mathrm{D}$. mccartyi cells were also collected from enrichment GW2.

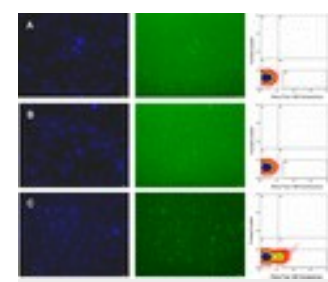

Figure 3. Fluorescence microscopy and flow cytometry analysis of enrichment GW2. Figure legend is the same as Figure 1

After amplifying four samples from duplicate cultures of enrichment GW2, the WGA DNA from $10^{6}$ and $10^{5}$ sorted cells were respectively hybridized onto microarrays for comparative genomics. Consistent with previous results with this enrichment,(30) the WGA DNA hybridized mostly to probes targeting strain 195 (Figure 4). Surprisingly, the average signal intensity of positive probe sets from the amplified samples with $10^{6}$ and $10^{5}$ cells was relatively low at 223 and 101, respectively. When compared against the gDNA results, only a $2.7 \%$ discrepancy in present/absent calls for probe sets (out of a total of 4161 ) was found in the sample with $10^{6}$ cells. As expected, a larger discrepancy of $9.0 \%$ was found in the sample prepared with $10^{5}$ cells. The overall genome structure of $D$. mccartyi in GW2 was preserved in the amplified DNA that started from $10^{6}$ cells, and the strain 195 genes that are absent in GW2 are concentrated within the integrated elements (IEs) and high-plasticity regions (HPRs) (Figure 4 ). In contrast, the amplified DNA originating from $10^{5}$ cells was unable to clearly resolve this genome profile (Figure 4 ), confirming that at least $1 \mathrm{ng}$ of starting DNA is necessary for high-fidelity amplification. The genes near genome position 1100 that were under-represented when $10^{6}$ strain 195 cells were amplified were absent in both the unamplified gDNA and amplified DNA of GW2, while genes near position 1400 were present in both types of GW2 samples. This verifies multiple amplifications are effective in both pure and enrichment cultures to minimize biases in regions that are difficult to amplify.

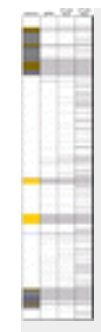

Figure 4. Microarray results from hybridization of gDNA (original data from Men et al.(30)) and WGA DNA from $10^{6}$ and $10^{5}$ cells of enrichment GW2. Each column represents a sample as indicated on the top and each row represents a gene of strain 195 where all the genes that are targeted by the microarrays (except a small number that tends to cross hybridize to nonspecific DNA) are depicted and arranged according to their location in the genome from top to bottom starting from DET0001. Genes that are considered present are colored white and gray indicates those that are absent. The "genome" column on the left depicts 
annotations that are of interest, including the two HPRs (dark shade), IEs I to IX (orange), putative RDases (dark blue), and the pce $A$ and $t c e A$ genes (purple).

When $10^{6} D$. mccartyi cells from the more dilute enrichment AD14 were sorted, amplified and hybridized onto microarrays, only $0.96 \%$ of the probe sets showed discrepancy when compared to results from direct hybridization of the gDNA (Figure 5), and the average signal intensity of 3229 for the positive probe sets was higher than enrichment GW2. Inspection of the microarray data showed that the genomes of the $D$. mccartyi population in this enrichment were similar to strain VS and that the WGA DNA essentially reproduced the overall genome structure of the gDNA (Figure $\underline{5}$ ).

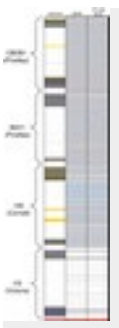

Figure 5. Microarray results from hybridization of gDNA and WGA DNA from $10^{6}$ cells of enrichment AD14. The figure legend is the same as Figure 4 except genes in the four sequenced genomes (marked on the left along with the subgroup classification and separated by a red horizontal line) are shown and the color light blue is used to indicate genes that are present and targeted by the same probe sets as genes in strain VS. In the "genome" column, the two HPRs of each strain (dark shade), IEs (I to IX) of strain 195 and IEs (I, III and VII) of strain CBDB1 (orange), putative RDases (dark blue), and the pceA, tce A, vcrA, and $b v c A$ genes (purple) are included.

\section{Genomic Analysis of D. mccartyi in Enrichment AD14}

The genomes of the D. mccartyi population in enrichment AD14 have not been previously analyzed. Using data from the amplified DNA, 82.4\% of genes in strain VS are present in the D. mccartyi population in enrichment AD14, and the $11.3 \%$ of strain VS genes that were not detected are located within the two previously defined HPRs near the origin of replication with the rest evenly distributed across the genome (Figure $\underline{5}$ ). Of the strain VS genes that are absent in the enrichment, over half of them are annotated to encode hypothetical proteins and reductive dehalogenases (RDases) or gene products that are related to RDases (Table S2 of the SI). Seventeen of the 101 RDases in the four sequenced genomes were detected in the enrichment (Table S3 of the SI), including the functionally characterized $t c e A(38)$ and $v c r A(39)$ genes. Using a previously published qPCR method, (32) quantification of the tceA, vcrA and D. mccartyi $16 \mathrm{~S}$ rRNA genes of the enrichment gDNA indicated that the ratio of tceA and $v c r A$ genes is $1: 1.6$ and the sum of these two genes closely match the quantity of the 16S rRNA gene, which is a single copy gene in the sequenced genomes.(16-18) Subsequently, using a dilution-to-extinction technique, Wang et al.(31) isolated two separate $D$. mccartyi strains from this enrichment; one that carries the tce $A$ gene and the other with the $v c r A$ gene. The comparative genomic results suggest that these two $D$. mccartyi strains share the core-genome of strain VS (Figure $\underline{5}$ ) and that the 16S rRNA gene sequences of the individual strains are identical over 1353 bases with only a single mismatch with strain VS.(31) 
The number of genes originally identified in D. mccartyi strains other than VS that were detected in the AD14 enrichment is relatively small with only 165 probe sets identified as positive (Table S4 of the SI). The majority of these probe sets (144) match genes of strain 195 and 42 of them are associated with hypothetical proteins. Interestingly, 29 of the detected strain 195 genes have similar functional annotations to genes in strain VS that were deemed absent in the enrichment, suggesting sequence divergence for these genes.

\section{Discussion}

FACS is a promising approach to isolate or enrich a subpopulation within a complex microbial community for genetic or genomic characterization. $(20,21,24,25)$ This method is especially suited to target uncultured microbes or those that are culturable but are challenging to isolate in sufficient quantities for sequencing. D. mccartyi is an ideal candidate for application of this technique as this anaerobic bacterium is fastidious in growth with a doubling time of $24 \mathrm{~h}$ or longer in isolation.(29) Even though D. mccartyi are functionally important in the bioremediation of chlorinated ethenes, their relative proportions in microbial communities from the environment tend to be low as demonstrated with field samples where $D$. mccartyi represented only $10^{-4} \%$ of the community.(40) Therefore, a significant amount of time and effort are often required to culture this bacterium in isolation or to enrich it sufficiently for downstream genomic analyses. All available $D$. mccartyi genomes have been obtained by isolating individual strains or through metagenomic sequencing of enrichment cultures.(13)

The goal of this study was to develop a FACS-WGA protocol applicable to $D$. mccartyi cells. Using a previously reported oligonucleotide probe, $(33)$ the fluorescence signals from $D$. mccartyi cells was detectable in microscopic observations and using a flow cytometer (Figures $\underline{1}$ and $\underline{3}$ ). Once cell sorting is enabled, the number of cells needed is driven by the objective of the investigation. Although a single cell can be sorted to analyze genotypic variation or ecotype, it remains challenging to accurately amplify DNA from a single cell to obtain complete genome coverage, and consequently, single cell amplification remains a research tool in the laboratory for scientific discovery. $(24,25$, $\underline{35,41-43)}$ When a larger number of cells are sorted and amplified, genome coverage improves significantly $\underline{22}$. 36) but the resulting pool of cells is representative of an averaged population. In addition to determining the number of cells that needs to be collected, choices are available for downstream genomic analysis. Sequencing is an option as well as application of microarrays for high-throughput comparative genomics. In this study, microarrays were chosen as the method of choice since this technique can be rapidly and economically applied in repeatable fashion to query genomes and a large number of samples can be processed and analyzed within a short period of time.

Although WGA is typically applied to obtain a sufficient quantity of DNA for genomic analysis after cell sorting, amplification biases are a major concern. Previous literature results $(22,35-37)$ have suggested that at least $1 \mathrm{ng}$ of DNA is required for high-fidelity amplification and results in this study corroborated this quantity. Based on results of strain 195 , we found that amplifying $10^{6}$ cells provided high quality genome coverage, with the observed intra- and intersample biases in amplification to be expected given that WGA involves random priming with some regions of the genome more difficult to amplify than others. $(25,35)$ Due to the inherent randomness in WGA and considering 
that complete genome coverage was important in this study, we performed four amplifications from $10^{6}$ cells for each culture analyzed in order to normalize any major amplification biases and to ensure near complete genome coverage.

When the FACS-WGA method was applied to enrichment cultures, the results from the amplified DNA closely matched those from the unamplified gDNA (Figures $\underline{4}$ and $\underline{5}$ ), demonstrating that cell sorting succeeded in isolating representative $D$. mccartyi cells and that the amplification procedure generated sufficient quantity and quality of DNA for high-fidelity detection. The microarray detection accuracy was $100 \%$ from the amplification of $10^{6}$ cells of strain 195 whereas in the enrichments, an error that ranges from 0.96 to $2.7 \%$ of the total probe sets was measured when compared against gDNA. Realistically, although minor errors are anticipated in the FACS-WGA process, the inaccuracies are minimized when a sufficient quantity of starting DNA is used in the amplification reactions. However, some variabilities were observed in the overall microarray signals from the two enrichment cultures tested in this study. This suggests some sorted $D$. mccartyi strains might be more challenging to amplify than others even though the same quantity of cells were used in the amplification step.

The purpose of developing the FACS-WGA method and coupling it with microarray analysis was to enable genomic characterization of minor and trace subpopulations within microbial communities. In enrichment AD14, both the tce $A$ and $v c r A$ genes, which encode enzymes for the reductive dechlorination of TCE to VC(38) and VC to ethene,(39) respectively, are present. Interestingly, by means of extensively laborious culture isolation, two separate $D$. mccartyi strains were recovered from the enrichment that were shown by qPCR of the tceA, vcrA and $16 \mathrm{~S}$ rRNA genes to represent the two dominant $D$. mccartyi strains in the AD14 enrichment.(31) The close match of the genomic results from the direct hybridization of gDNA with those of the FACS-WGA indicate that the FACSWGA method did not introduce significant biases to the comparative genomic analysis, suggesting that the result reflects the genome structures of the two dominant strains. This coupled with the broad specificity of the 16S rRNA FISH probe applied in the FACS technique, enabled multiple $D$. mccartyi strains to be captured from the microbial community and analyzed simultaneously.

The tce $A$ gene has previously been observed to be present in strains that are associated with two of the three designated $D$. mccartyi subgroups, Pinellas and Cornell. $(19,38,44)$ This study is the first to report a tce $A$ gene associated with the third D. mccartyi subgroup, Victoria. Recently, the three well-characterized RDases ( $p c e A, t c e A$, and $v c r A$ ) have also been reported in the genome of strain BTF08, which should enable all steps of reductive dechlorination from tetrachloroethene to ethene.(45) These results lend further evidence to the concept that horizontal gene transfer can confer dechlorinating functions broadly to $D$. mccartyi populations regardless of their phylogenetic affiliation. $(17,19)$ Previous comparative genomic analyses have shown that $D$. mccartyi genomes share a core genome and that most differences between strains occur within distinct HPRs.(17, 19) Interestingly, in the case of enrichment AD14, a higher number of genes (6.4\%) that do not match the strain VS genome were actually found to be distributed across the genome outside of the HPRs (Figure $\underline{5}$ ), whereas the overall core metabolic functions were present. 
In summary, FACS is a valuable tool to target and separate organisms of interests in microbial communities for downstream genomic analyses as part of the molecular toolkit to study environmental microbial communities, including those involved in bioremediation. In this study, as proof of concept, we demonstrated that it is feasible to couple FACS with WGA and microarray analysis to analyze D. mccartyi genomes within enrichment cultures. The successful separation and concentration of these fastidious bacteria facilitates genomic characterization, especially when analyzing samples collected directly from the environment where the functionally important $D$. mccartyi are often only a minor fraction of the microbial community.

\section{Acknowledgment}

We thank Hector Nolla and Alma Valeros for their expertise in flow cytometry and Denise Schichnes for her expertise in microscopy. This research was supported by the Strategic Environmental Research and Development Program (SERDP) through grant ER-1587, the National Science Foundation grant CBET-1336709, and the NIEHS Superfund Basic Research Project ES04705-19.

\section{References}

This article references 45 other publications.

1.

Moran, M. J.; Zogorski, J. S.; Squillace, P. J. Chlorinated solvents in groundwater of the United StatesEnviron. Sci. Technol. 2007, 41, 74- 81

[ACS Full Text $\odot$ ], [CAS]

2.

Löffler, F. E.; Edwards, E. A. Harnessing microbial activities for environmental cleanup Curr. Opin.

Biotechnol. 2006, 17, 274- 284

[Crossref], [PubMed], [CAS]

3.

Maphosa, F.; de Vos, W. M.; Smidt, H. Exploiting the ecogenomics toolbox for environmental diagnostics of organohaliderespiring bacteria Trends Biotechnol. 2010, 28, 308- 316

[Crossref], [PubMed], [CAS]

$\underline{4}$.

Krajmalnik-Brown, R.; Hölscher, T.; Thomson, I. N.; Saunders, F. M.; Ritalahti, K. M.; Löffler, F. E. Genetic identification of a putative vinyl chloride reductase in Dehalococcoides sp. strain BAV1 Appl. Environ. Microbiol. 2004, 70, 6347- 6351

[Crossref], [PubMed], [CAS]

$\underline{5 .}$

Waller, A. S.; Krajmalnik-Brown, R.; Löffler, F. E.; Edwards, E. A. Multiple reductive-dehalogenase-homologous genes are simultaneously transcribed during dechlorination by Dehalococcoides-containing cultures Appl. Environ.

Microbiol. 2005, 71, 8257- 8264

[Crossref], [PubMed], [CAS]

$\underline{6}$. 
Behrens, S.; Azizian, M. F.; McMurdie, P. J.; Sabalowsky, A.; Dolan, M. E.; Semprini, L.; Spormann, A. M.Monitoring abundance and expression of "Dehalococcoides" species chloroethene-reductive dehalogenases in a tetrachloroethenedechlorinating flow column Appl. Environ. Microbiol. 2008, 74, 5695- 5703

[Crossref], [ubMed], [도]

\section{7.}

Lee, P. K. H.; Macbeth, T. W.; Sorenson, K. S., Jr.; Deeb, R. A.; Alvarez-Cohen, L. Quantifying genes and transcripts to assess the in situ physiology of "Dehalococcoides" spp. in a trichloroethene-contaminated groundwater site Appl. Environ.

Microbiol. 2008, 74, 2728- 2739

[Crossref], [PubMed], [CAS]

$\underline{8}$.

Fennell, D. E.; Carroll, A. B.; Gossett, J. M.; Zinder, S. H. Assessment of indigenous reductive dechlorinating potential at a TCE-contaminated site using microcosms, polymerase chain reaction analysis, and site data Environ. Sci.

Technol. 2001, 35, 1830- 1839

[ACS Full Text $-\oplus$ ], [CAS]

$\underline{9}$.

Hendrickson, E. R.; Payne, J. A.; Young, R. M.; Starr, M. G.; Perry, M. P.; Fahnestock, S.; Ellis, D. E.;Ebersole, R. C. Molecular analysis of Dehalococcoides $16 \mathrm{~S}$ ribosomal DNA from chloroethene-contaminated sites throughout North America and Europe Appl. Environ. Microbiol. 2002, 68, 485-495

[Crossref], [PubMed], [CAS]

10.

van der Zaan, B.; Hannes, F.; Hoekstra, N.; Rijnaarts, H.; de Vos, W. M.; Smidt, H.; Gerritse, J. Correlation of Dehalococcoides $16 \mathrm{~S}$ rRNA and chloroethene-reductive dehalogenase genes with geochemical conditions in chloroethene-contaminated groundwater Appl. Environ. Microbiol. 2010, 76, 843-850

[Crossref], [PubMed], [AS]

11.

Hug, L. A.; Edwards, E. A. Diversity of reductive dehalogenase genes from environmental samples and enrichment cultures identified with degenerate primer PCR screens Front. Microbiol. 2013, 4, 341

[Crossref], [PubMed], [CAS]

12.

Hug, L. A.; Maphosa, F.; Leys, D.; Löffler, F. E.; Smidt, H.; Edwards, E. A.; Adrian, L. Overview of organohalide-respiring bacteria and a proposal for a classification system for reductive dehalogenasesPhilos. Trans. R. Soc.,

B 2013, 368, 20120322

[Crossref], [PubMed], [CAS]

13.

Richardson, R. E. Genomic insights into organohalide respiration Curr. Opin. Biotechnol. 2013, 24, 498-505

[Crossref], [PubMed], [CAS]

14.

Vieites, J. M.; Guazzaroni, M. E.; Beloqui, A.; Golyshin, P. N.; Ferrer, M. Metagenomics approaches in systems microbiology FEMS Microbiol. Rev. 2009, 33, 236- 255

[Crossref], [PubMed], [CAS]

15.

He, Z.; Gentry, T. J.; Schadt, C. W.; Wu, L.; Liebich, J.; Chong, S.

C.; Huang, Z.; Wu, W.; Gu, B.; Jardine, P.;Criddle, C.; Zhou, J. GeoChip: A comprehensive microarray for investigating biogeochemical, ecological and environmental processes ISME J. 2007, 1, 67- 77

[Crossref], [PubMed], [CAS]

16. 
Kube, M.; Beck, A.; Zinder, S. H.; Kuhl, H.; Reinhardt, R.; Adrian, L. Genome sequence of the chlorinated compoundrespiring bacterium Dehalococcoides species strain CBDB1 Nat. Biotechnol. 2005, 23, 1269-1273

[Crossref], [PubMed], [CAS]

17.

McMurdie, P. J.; Behrens, S. F.; Müller, J. A.; Göke, J.; Ritalahti, K.

M.; Wagner, R.; Goltsman, E.;Lapidus, A.; Holmes, S.; Löffler, F. E.; Spormann, A. M. Localized plasticity in the streamlined genomes of vinyl chloride respiring Dehalococcoides PLoS Genet. 2009, 5, e1000714

[Crossref], [PubMed], [CAS]

18.

Seshadri, R.; Adrian, L.; Fouts, D. E.; Eisen, J. A.; Phillippy, A. M.; Methe, B. A.; Ward, N. L.; Nelson, W. C.;Deboy, R. T.; Khouri, H. M.; Kolonay, J. F.; Dodson, R. J.; Daugherty, S. C.; Brinkac, L. M.; Sullivan, S. A.; Madupu, R.; Nelson, K. E.; Kang, K. H.; Impraim, M.; Tran, K.; Robinson, J. M.; Forberger, H. A.;Fraser, C. M.; Zinder, S. H.; Heidelberg, J.

F. Genome sequence of the PCE-dechlorinating bacteriumDehalococcoides ethenogenes Science 2005, 307, 105- 108 [Crossref], [PubMed], [CAS]

19.

Lee, P. K. H.; Cheng, D.; Hu, P.; West, K. A.; Dick, G. J.; Brodie, E. L.; Andersen, G. L.; Zinder, S. H.; He, J.;AlvarezCohen, L. Comparative genomics of two newly isolated Dehalococcoides strains and an enrichment using a genus microarray ISME J. 2011, 5, 1014- 1024

[Crossref], [PubMed], [CAS]

$\underline{20 .}$

Czechowska, K.; Johnson, D. R.; van der Meer, J. R. Use of flow cytometric methods for single-cell analysis in environmental microbiology Curr. Opin. Microbiol. 2008, 11, 205- 212

[Crossref], [PubMed], [CAS]

$\underline{21 .}$

Müller, S.; Nebe-von-Caron, G. Functional single-cell analyses: Flow cytometry and cell sorting of microbial populations and communities FEMS Microbiol. Rev. 2010, 34, 554- 587

[PubMed], [CAS]

$\underline{22 .}$

Kalyuzhnaya, M. G.; Zabinsky, R.; Bowerman, S.; Baker, D. R.; Lidstrom, M. E.; Chistoserdova, L.Fluorescence in situ hybridization-flow cytometry-cell sorting-based method for separation and enrichment of type I and type II methanotroph populations Appl. Environ. Microbiol. 2006, 72, 4293- 4301

[Crossref], [PubMed], [CAS]

$\underline{23 .}$

Woyke, T.; Xie, G.; Copeland, A.; González, J. M.; Han, C.; Kiss, H.; Saw, J. H.; Senin, P.; Yang, C.;Chatterji, S.; Cheng, J. F.; Eisen, J. A.; Sieracki, M. E.; Stepanauskas, R. Assembling the marine metagenome, one cell at a time PLoS One 2009, 4, e5299

[Crossref], [PubMed], [CAS]

24.

Lasken, R. S. Genomic sequencing of uncultured microorganisms from single cells Nat. Rev. Microbiol.2012, 10, 631- 640 [Crossref], [PubMed], [CAS]

$\underline{25}$.

Blainey, P. C. The future is now: Single-cell genomics of bacteria and archaea FEMS Microbiol. Rev. 2013,37, 407- 427 [Crossref], [PubMed], [CAS]

26.

Löffler, F. E.; Yan, J.; Ritalahti, K. M.; Adrian, L.; Edwards, E. A.; Konstantinidis, K. T.; Müller, J. A.;Fullerton, H.; Zinder, S. H.; Spormann, A. M. Dehalococcoides mccartyi gen. nov., sp. nov., obligate organohalide-respiring anaerobic bacteria, 
relevant to halogen cycling and bioremediation, belong to a novel bacterial class, Dehalococcoidetes classis nov., within the phylum Chloroflexi Int. J. Syst. Evol. Microbiol.2013, 63, 625- 635

[Crossref], [PubMed], [CAS]

27.

Maymó-Gatell, X.; Chien, Y. T.; Gossett, J. M.; Zinder, S. H. Isolation of a bacterium that reductively dechlorinates tetrachloroethene to ethene Science 1997, 276, 1568- 1571

[Crossref], [PubMed], [CAS]

$\underline{28 .}$

He, J.; Holmes, V. F.; Lee, P. K. H.; Alvarez-Cohen, L. Influence of vitamin B12 and cocultures on the growth of Dehalococcoides isolates in defined medium Appl. Environ. Microbiol. 2007, 73, 2847- 2853

[Crossref], [PubMed], [CAS]

$\underline{29}$.

He, J.; Ritalahti, K. M.; Yang, K. L.; Koenigsberg, S. S.; Löffler, F. E. Detoxification of vinyl chloride to ethene coupled to growth of an anaerobic bacterium Nature 2003, 424, 62- 65

[Crossref], [PubMed], [CAS]

$\underline{30 .}$

Men, Y.; Lee, P. K. H.; Harding, K. C.; Alvarez-Cohen, L. Characterization of four TCE-dechlorinating microbial enrichments grown with different cobalamin stress and methanogenic conditions Appl. Microbiol. Biotechnol. 2013, 97, 6439- 6450 [Crossref], [PubMed], [CAS]

$\underline{31 .}$

Wang, S.; He, J. Dechlorination of commercial PCBs and other multiple halogenated compounds by a sediment-free culture containing Dehalococcoides and Dehalobacter Environ. Sci. Technol. 2013, 47,10526- 10534

[ACS Full Text $\odot$ ], [CAS]

$\underline{32 .}$

Holmes, V. F.; He, J.; Lee, P. K. H.; Alvarez-Cohen, L. Discrimination of multiple Dehalococcoides strains in a trichloroethene enrichment by quantification of their reductive dehalogenase genes Appl. Environ.

Microbiol. 2006, 72, 5877- 5883

[Crossref], [PubMed], [CAS]

$\underline{33}$.

Yang, Y.; Zeyer, J. Specific detection of Dehalococcoides species by fluorescence in situ hybridization with 16S rRNAtargeted oligonucleotide probes Appl. Environ. Microbiol. 2003, 69, 2879- 2883

[Crossref], [PubMed], [소]

34.

Amann, R.; Ludwig, W.; Schleifer, K. Phylogenetic identification and in situ detection of individual microbial cells without cultivation Microbiol. Rev. 1995, 59, 143- 169

[PubMed], [CAS]

$\underline{35 .}$

Binga, E. K.; Lasken, R. S.; Neufeld, J. D. Something from (almost) nothing: The impact of multiple displacement amplification on microbial ecology ISME J. 2008, 2, 233- 241

[Crossref], [PubMed], [CAS]

$\underline{36 .}$

Chen, Y.; Dumont, M. G.; Neufeld, J. D.; Bodrossy, L.; Stralis-Pavese, N.; McNamara, N. P.; Ostle, N.;Briones, M. J. I.; Murrell, J. C. Revealing the uncultivated majority: Combining DNA stable-isotope probing, multiple displacement amplification and metagenomic analyses of uncultivated Methylocystis in acidic peatlands Environ.

Microbiol. 2008, 10, 2609- 2622

[Crossref], [PubMed], [CAS] 
$\underline{37 .}$

Dean, F. B.; Hosono, S.; Fang, L.; Wu, X.; Faruqi, A. F.; Bray-

Ward, P.; Sun, Z.; Zong, Q.; Du, Y.; Du, J.;Driscoll, M.; Song, W.; Kingsmore, S. F.; Egholm, M.; Lasken, R. S. Comprehensive human genome amplification using multiple displacement amplification Proc. Natl. Acad. Sci. U.S.A. 2002, 99, 5261-5266 [Crossref], [PubMed], [CAS]

38.

Magnuson, J. K.; Romine, M. F.; Burris, D. R.; Kingsley, M. T. Trichloroethene reductive dehalogenase fromDehalococcoides ethenogenes: Sequence of tceA and substrate range characterization Appl. Environ.

Microbiol. 2000, 66, 5141- 5147

[Crossref], [PubMed], [CAS]

$\underline{39 .}$

Müller, J. A.; Rosner, B. M.; von Abendroth, G.; Meshulam-Simon, G.; McCarty, P. L.; Spormann, A. M.Molecular identification of the catabolic vinyl chloride reductase from Dehalococcoides sp. strain VS and its environmental distribution Appl. Environ. Microbiol. 2004, 70, 4880- 4888

[Crossref], [PubMed], [CAS]

40.

Ritalahti, K. M.; Hatt, J. K.; Lugmayr, V.; Henn, K.; Petrovskis, E. A.; Ogles, D. M.; Davis, G. A.;Yeager, C. M.; Lebrón, C. A.; Löffler, F. E. Comparing on-site to off-site biomass collection forDehalococcoides biomarker gene quantification to predict in situ chlorinated ethene detoxification potentialEnviron. Sci. Technol. 2010, 44, 5127- 5133

[ACS Full Text $\odot$ ], [CAS]

41.

Raghunathan, A.; Ferguson, H. R., Jr.; Bornarth, C. J.; Song, W.; Driscoll, M.; Lasken, R. S. Genomic DNA amplification from a single bacterium Appl. Environ. Microbiol. 2005, 71, 3342- 3347

[Crossref], [PubMed], [CAS]

42.

Rodrigue, S.; Malmstrom, R. R.; Berlin, A. M.; Birren, B. W.; Henn, M. R.; Chisholm, S. W. Whole genome amplification and de novo assembly of single bacterial cells PLoS One 2009, 4, e6864

[Crossref], [PubMed], [CAS]

43.

Zhang, K.; Martiny, A. C.; Reppas, N. B.; Barry, K. W.; Malek, J.; Chisholm, S. W.; Church, G. M.Sequencing genomes from single cells by polymerase cloning Nat. Biotechnol. 2006, 24, 680-686

[Crossref], [PubMed], [CAS]

$\underline{44 .}$

He, J.; Sung, Y.; Krajmalnik-Brown, R.; Ritalahti, K. M.; Löffler, F. E. Isolation and characterization ofDehalococcoides sp. strain FL2, a trichloroethene (TCE)- and 1,2-dichloroethene-respiring anaerobeEnviron. Microbiol. 2005, 7, $1442-1450$ [Crossref], [PubMed], [CAS]

45.

Pöritz, M.; Goris, T.; Wubet, T.; Tarkka, M. T.; Buscot, F.; Nijenhuis, I.; Lechner, U.; Adrian, L. Genome sequences of two dehalogenation specialists-Dehalococcoides mccartyi strains BTF08 and DCMB5 enriched from the highly polluted Bitterfeld region FEMS Microbiol. Lett. 2013, 343, 101- 104

[Crossref], [PubMed], [CAS] 